\title{
Acidic Ribosomal Proteins and Histone H3 from Leishmania Present a High Rate of Divergence
}

\author{
Ysabel Montoya ${ }^{+}$, Carlos Padilla, Maxy De Los Santos, Teresa Barreto, \\ Douglas Barker*, Carlos Carrillo**
}

\author{
Instituto Nacional de Salud, Capac Yupanqui 1400, Lima 11, Peru *University of Cambridge, UK \\ **Universidad Peruana Cayetano Heredia, Honorio Delgado, Lima 31, Peru
}

Another additional peculiarity in Leishmania will be discussed about of the amino acid divergence rate of three structural proteins: acidic ribosomal $P 1$ and $P 2 b$ proteins, and histone $H 3$ by using multiple sequence alignment and dendrograms.

These structural proteins present a high rate of divergence regarding to their homologous protein in Trypanosoma cruzi. At this regard, L. (V.) peruviana $P 1$ and T. cruzi $P 1$ showed $57.4 \%$ of divergence rate. Likewise, L. (V.) braziliensis histone $\mathrm{H} 3$ and acidic ribosomal $\mathrm{P} 2$ protein exhibited $31.8 \%$ and $41.7 \%$ respectively of rate of divergence in comparison with their homologous in $\mathrm{T}$. cruzi.

Key words: Leishmania - acidic ribosomal proteins - histone $\mathrm{H} 3$

Acidic ribosomal proteins known as $\mathrm{P}$ (phospho) proteins are present in the ribosomes belonging to the eubacteria, archaebacteria and eukaryotes. These conserved proteins are placed in the large ribosomal sub-unit forming a highly flexible lateral projection referred as the ribosomal stalk (Wittman 1983, Lake 1985). The stalk is involved in the interaction between the translation factors and the ribosome during protein synthesis (Shimmin et al. 1989).

Acidic ribosomal P1 protein in trypanosomatids had not been reported in the Leishmania genus previous to this work. However, the $\mathrm{P} 0$ and $\mathrm{P} 2$ ribosomal proteins had been characterised in $L$. infantum (Soto et al. 1995a, b), L. chagasi (Skeiky et al. 1994) and L. donovani (Kunz et al. 1993). Moreover, Trypanosoma cruzi acidic ribosomal protein belong to a gene family found in multiple copies (TcP0, TcP1, TcP2a and TcP2b). The P

Y Montoya received partial support from a re-entry grant provided by the UNDP/World Bank/WHO Special Programme for Research and Training in Tropical Diseases. This work also received partial financial support from the STD-3 Programme of the European Community, contract $\mathrm{TS} 3 *$ CT92-0123.

${ }^{+}$Corresponding author. Fax: +511-471.2529. E-mail: ymontoya@ins.sld.pe

Received 13 April 2000

Accepted 15 May 2000 proteins are important antigens that generate humoral response in leishmaniasis, Chagas disease and systemic lupus erythematosus (Elkon et al. 1986).

Despite of presenting histones, the trypanosomatids do not condense their chromatin during mitosis (Solari 1980). Likewise, trypanosomatids histone $\mathrm{H} 3$ presents an extremely divergent N-terminal domain (Galanti et al. 1998). Another important peculiarity is that trypanosomatids histone $\mathrm{H} 3$ lacks of the amino acids sequence KSTGGKA at the N-terminal end, which is present in the consensus sequence of higher eukaryotes (Wells 1986).

Three clones from $L$. (V.) peruviana (T26-U3) and $L$. (V.) braziliensis cDNA libraries (T166-U19 and T166-M49) were previously selected by their sero-reactivity with leishmaniasis patients (Montoya 1993). The DNA insert corresponding to each of them was amplified and sub-cloned in pUC18/EcoRI/BAP (Pharmacia) plasmid vector.

After sequence analysis, the T26-U3 clone was identified as a $L$. $(V$.) peruviana acidic ribosomal protein $\mathrm{P} 1$ being referred as LpP1. The clone T166U19 was recognised as a $L$. (V.) braziliensis acidic ribosomal protein $\mathrm{P} 2 \mathrm{~b}$ and referred as LbP2b. Finally, the T166-M49 clone was identified as a $L$. (V.) braziliensis histone $\mathrm{H} 3$ being referred as $\mathrm{LbH} 3$.

The deduced amino acid sequence of the LpP1 had a divergence of $57.4 \%$ in comparison with $T$. cruzi $\mathrm{P} 1$ protein (TcP1). LpP2b showed $17.9 \%$ of divergence with the $L$. donovani ribosomal $\mathrm{P} 2$ protein (LdP2) and $41.7 \%$ of divergence with their 
homologous in $T$. cruzi $(\mathrm{TcP} 2)$. Finally, $\mathrm{LbH} 3$ presented $17.7 \%$ and $31.8 \%$ of divergence in comparison with the sequences of $L$. infantum (LiH3) and $T$. cruzi $(\mathrm{TcH} 3)$ respectively (Table).

Multiple alignment of sequences was performed using CLUSTALW 1.5 program and also for establishing the phylogenetic relationships among these proteins was used PHYLIP 3.5c program. For this purpose, eight eukaryotic P2 proteins (Fig. 1) and six histones H3 (Fig. 2) were included. The bootstrap values (bv) were obtained from a consensus tree based on 100 randomly generated trees.

The genetic distances were obtained by Kimura method showing a higher value between $\mathrm{LpP} 1$ and TcP1 (0.83902), in contrast with the value observed

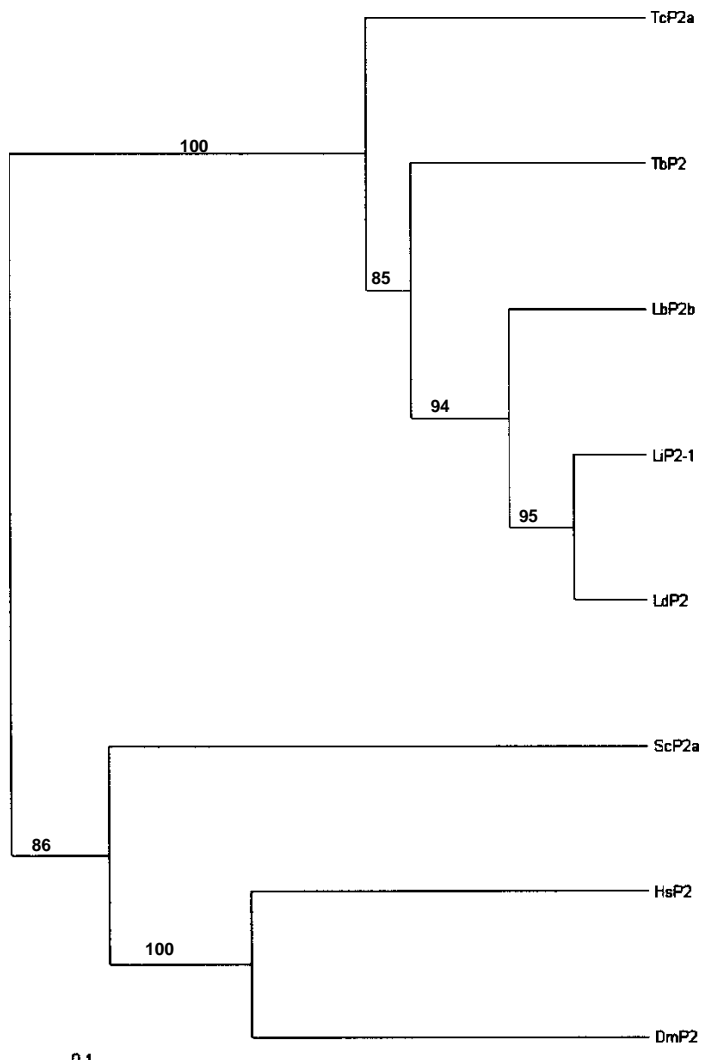

Fig. 1: phylogenetic analysis of $\mathrm{P} 2$ acidic ribosomal proteins. Phylogenetic relationships were inferred by using PHYLIP 3.5c. Evolutionary distance matrices, generated by PROTDIST, were determined by using Kimura method. Matrices were used to construct dendrograms using KITSCH program. Bootstrap values were obtained for a consense tree based on 100 randomly generated trees by using SEQBOOT and CONSENSE in the same package, based on the amino acid sequences of $8 \mathrm{P} 2$ protein: Leishmania brasiliensis (LbP2b), L. donovani (LdP2), L. infantum (LiP2-1), Trypanosoma cruzi (TcP2a), T. brucei brucei (HsP2). Phylogenetic distances scale, is shown in left botton corner. Value indicated in each nodes represent bootstrap value in the respective branch.

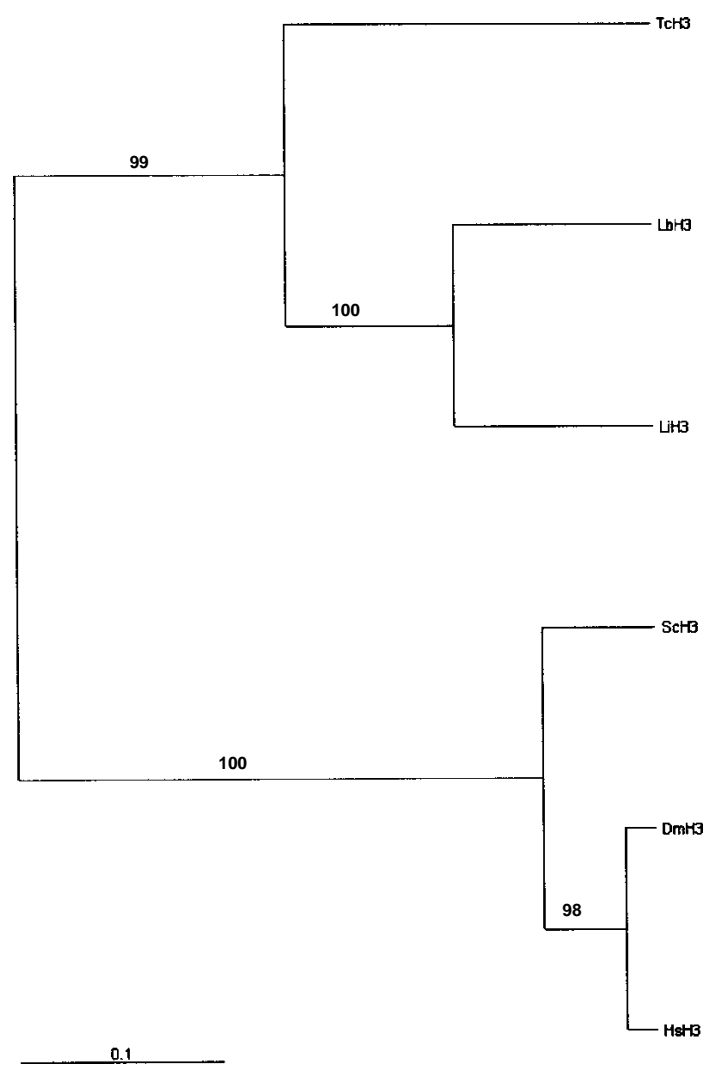

Fig. 2: phylogenetic analysis of histone $\mathrm{H} 3$ proteins based on the amino acid sequences of six histone $\mathrm{H} 3$ proteins (b): Leishmania brasiliensis (LbH3), L. infantum (LiH3), Trypanosoma cruzi $(\mathrm{TcH} 3)$, Saccharomyces cerevisiae $(\mathrm{ScH} 3)$ and Homo sapiens (HsH3). Phylogenetic distances scale is shown in left botton corner. Value indicated in each nodes represent bootstrap value in the respective branch.

between LbP2b and TcP2 (0.39811), and also the value obtained between $\mathrm{LbH} 3$ and TcH3 (0.38273) (Table). Likewise, LbP2b was very related to $L$. donovani $\mathrm{P} 2$ protein whose phylogenetic distance was 0.18489 and their divergence rate was $17.9 \%$. Therefore, our results show that these structural proteins present a high rate of divergence regarding to their homologous protein with $T$. cruzi.

Our results based on the analysis sequence of the $L .(V$.) braziliensis histone $\mathrm{H} 3$ and $L$. $(V$.) peruviana $\mathrm{P} 2$ proteins indicate that Leishmania is monophyletic. Trypanosomatids P2 clade was separated from the higher eukaryotes clade and sustained with a high bootstrap value (100\%) (Fig. 1). In a similar way, trypanosomatids histone $\mathrm{H} 3$ clade was separated from the higher eukaryotes clade with a high bootstrap value (99\%) (Fig. 2).

Histones $\mathrm{H} 3$ are structural proteins considered as one of the most conserved proteins. For example, the divergence rate reported previously between 
TABLE

The percentage of amino acid divergence (below diagonal) and phylogenetic distances calculated by Kimura method (above diagonal) among trypanosomatids acidic ribosomal $\mathrm{P}$ and histone $\mathrm{H} 3$ protein. In parenthesis number of conservatives changes and no conservatives changes

\begin{tabular}{|c|c|c|c|c|c|c|c|c|}
\hline & LpP1 & TcP1 & ScP1a & ScP1b & DmP1 & HsP1 & & \\
\hline LpP1 & & 0.83902 & 1.05672 & 1.09255 & 0.92079 & 0.93689 & & \\
\hline TcP1 & $57.4 \%(20 / 32)$ & & 0.87003 & 0.79851 & 1.01262 & 0.71531 & & \\
\hline ScP1a & $62.5 \%(20 / 36)$ & $61.5 \%(15 / 35)$ & & 0.64449 & 0.87895 & 0.98490 & & \\
\hline ScP1b & $63.4 \%(24 / 32)$ & $58.1 \%(16 / 31)$ & $46.8 \%(20 / 25)$ & & 1.03140 & 1.08584 & & \\
\hline DmP1 & $57.9 \%(24 / 32)$ & $86.2 \%(28 / 31)$ & $57.0 \%(22 / 33)$ & $61.4 \%(28 / 32)$ & & 0.58440 & & \\
\hline HsP1 & $59.8 \%(21 / 35)$ & $50.9 \%(17 / 33)$ & $61.5 \%(20 / 38)$ & $64.1 \%(24 / 37)$ & $44.0 \%(18 / 27)$ & & & \\
\hline & $\mathrm{LbP} 2 b$ & $\mathrm{LdP} 2$ & LiP2-1 & $\mathrm{TbP} 2$ & $\mathrm{TcP} 2 \mathrm{a}$ & $\mathrm{ScP} 2 \mathrm{a}$ & DmP2 & $\mathrm{HsP} 2$ \\
\hline LbP2b & & 0.18489 & 0.20764 & 0.40351 & 0.39811 & 0.73231 & 0.96413 & 0.89606 \\
\hline LdP2 & $\mathbf{1 7 . 9 \%}(10 / 7)$ & & 0.10209 & 0.30449 & 0.36431 & 0.82614 & 0.75820 & 0.86709 \\
\hline LiP2-1 & $18.9 \%(12 / 7)$ & $10.4 \%(3 / 7)$ & & 0.32018 & 0.43335 & 0.88412 & 0.88412 & 0.90973 \\
\hline TbP2 & $43.1 \%(13 / 17)$ & $37.9 \%(11 / 13)$ & 39.3\% (11/14) & & 0.41084 & 0.91716 & 1.06912 & 1.03662 \\
\hline TcP2a & $41.7 \%(10 / 20)$ & $\mathbf{4 0 . 0 \%}(9 / 19)$ & $44.0 \%(8 / 24)$ & $38.9 \%(10 / 22)$ & & 1.08439 & 1.04208 & 1.18399 \\
\hline ScP2a & $55.3 \%(16 / 28)$ & $57.2 \%(18 / 30)$ & $59.7 \%(20 / 30)$ & $62.4 \%(18 / 33)$ & $65.2 \%(21 / 36)$ & & 0.93983 & 0.67660 \\
\hline DmP2 & $64.2 \%(23 / 29)$ & $58.3 \%(17 / 28)$ & $61.7 \%(18 / 32)$ & $68.3 \%(19 / 37)$ & $64.7 \%(19 / 39)$ & $60.3 \%(22 / 32)$ & & 0.56563 \\
\hline HsP2 & $60.5 \%(25 / 27)$ & $59.7 \%(21 / 30)$ & $60.5 \%(24 / 29)$ & $64.2 \%(25 / 33)$ & $67.5 \%(25 / 37)$ & $52.9 \%(19 / 26)$ & $48.4 \%(14 / 27)$ & \\
\hline & $\mathrm{LbH} 3$ & LiH3 & $\mathrm{TcH} 3$ & $\mathrm{ScH} 3$ & HsH3 & & & \\
\hline LbH3 & & 0.19402 & 0.38273 & 0.60450 & 0.60450 & & & \\
\hline LiH3 & $\mathbf{1 7 . 7 \%}(13 / 9)$ & & 0.33693 & 0.54670 & 0.57849 & & & \\
\hline ТсH3 & $\mathbf{3 1 . 8 \%}(17 / 22)$ & $29.5 \%(12 / 23)$ & & 0.72004 & 0.75840 & & & \\
\hline ScH3 & $45.3 \%(15 / 39)$ & $43.1 \%(10 / 39)$ & $49.6 \%(15 / 46)$ & & 0.10249 & & & \\
\hline HsH3 & $42.3 \%(17 / 37)$ & $44.5 \%(12 / 39)$ & $51.1 \%(16747)$ & $9.6 \%(4 / 9)$ & & & & \\
\hline
\end{tabular}

Number accessions of P1 proteins: LpP1, Leishmania peruviana (AF045249); TcP1, Trypanosoma cruzi (X65025); ScP1a, Saccharomyces cerevisiae (PO5318); ScP1b, S. cerevisiae (P10622); DmP1, Drosophila melanogaster (Y00504) and HsP1, Homo sapiens (NM001003). Number accessions of P2 proteins: LbP2b, L. braziliensis (AF045020); LdP2, L. donovani (O43940); LiP2-1, L. infantum (Q06383); TcP2a, T. cruzi (P23632); TbP2, T. brucei brucei (P51408); ScP2a, S. cerevisiae (PO5319) and HsP2, H. sapiens (NM001004). Number accessions of histone H3: LbH3, L. braziliensis (AF044682); LiH3, L. infantum (P40285); TcH3, T. cruzi (AAA61706); ScH3, S. cerevisiae (P02303) and $\mathrm{HsH} 3$, H. sapiens (NP 005315). 
human and Saccharomyces histone $\mathrm{H} 3$ was just 9.6\% (Table). However, in Leishmania a high rate of divergence at the $\mathrm{N}$-terminal end and the separation of the trypanosomatids clade out of the higher eukaryotes clade is reported here (Fig. 2). This changes in the N-terminal end could contribute in the lost of chromatin condensation.

The divergence rate observed between $\mathrm{P} 1$ proteins (LpP1 and TcP1 57.4\%) showed a value fluctuating in the range of the higher eukaryotes (44\% to $64.1 \%$ ) (Table). The low number of sequences reported to this protein did not allow establish the phylogenetic relationship among these proteins.

Further studies should be performed to relate the high divergence rate detected in these parasites with their functional implications during the translation.

\section{ACKNOWLEDGEMENT}

To Dr Jr Stevens for helping with the manuscript.

\section{REFERENCES}

Elkon K, Skelly S, Parnassa A, Moller W, Danho W, Weissbach H, Brot N 1986. Identification and chemical synthesis of a ribosomal protein antigenic determinant in systemic lupus erythematosus. Proc Natl Acad Sci USA 83: 7419-7423.

Galanti N, Galindo M, Sabaj V, Espinoza I, Toro G 1998. Histone genes in Trypanosomatids. Parasitol Today 14: 64-70.

Kunz S, Mueller N, Seebeckt 1993. Genbank direct sub- mission No. 043940.

Lake J 1985. Evolving ribosome structure: domains in archaebacteria, eubacteria, eocytes and eukaryotes. Ann Rev Biochem 54: 507-530.

Montoya Y 1993. Molecular Analysis of Antigen Genes in Peruvian Leishmania, PhD Thesis, Cambridge.

Shimmin LC, Ramirez C, Matheson AT, Dennis P 1989. Sequence alignment and evolutionary comparison of the L10 equivalent and L12 equivalent ribosomal proteins from archaebacteria, eubacteria, and eucayotes. J Mol Evol 29: 448-462.

Skeiky YA, Benson DR, Elwasila M, Badaro R, Burns J, Reed SG 1994. Antigens shared by Leishmania species and Trypanosoma cruzi: immunological comparison of the acidic ribosomal $\mathrm{P} 0$ proteins. Infec Immun 62: 1643-1651.

Solari AJ 1980. The 3-dimensional fine structure of mitotic spindle in Trypanosoma cruzi. Chromosome 78: 239-255.

Soto M, Requena JM, Quijada L, Angel SO, Gomez LC, Guzman F, Patarroyo ME, Alonso C 1995a. During active viscerocutaneous leishmaniasis the anti-P2 humoral response is specifically triggered by the parasite P proteins. Clin Exp Immunol 100: 246-252.

Soto M, Requena JM, Garcia M, Gomez LC, Navarrete I, Alonso C 1995b. Identification of the Leishmania infantum $\mathrm{P} 0$ ribosomal protein epitope in canine visceral leishmaniasis. Immunol Lett 48: 23-28.

Wells D 1986. Compilation analysis of histones and histone genes. Nucleic Acids Research 14: 119-149.

Wittman HG 1983. Architecture of prokaryotic ribosomes. Ann Rev Biochem 52: 35-65. 